\title{
A Reconstruction Filter for Saturated Accelerometer Signals Due to Insufficient FSR in Foot-mounted Inertial Navigation System
}

This paper was downloaded from TechRxiv (https://www.techrxiv.org).

\section{LICENSE}

CC BY 4.0

SUBMISSION DATE / POSTED DATE

$05-10-2021 / 18-10-2021$

\section{CITATION}

Jao, Chi-Shih; Shkel, Andrei M. (2021): A Reconstruction Filter for Saturated Accelerometer Signals Due to Insufficient FSR in Foot-mounted Inertial Navigation System. TechRxiv. Preprint.

https://doi.org/10.36227/techrxiv.16745839.v1

$\mathrm{DOI}$

10.36227/techrxiv.16745839.v1 


\title{
A Reconstruction Filter for Saturated Accelerometer Signals Due to Insufficient FSR in Foot-mounted Inertial Navigation System
}

\author{
Chi-Shi Jao, Student Member, IEEE, and Andrei M. Shkel, Fellow, IEEE
}

\begin{abstract}
In pedestrian inertial navigation, one possible placement of Inertial Measurement Units (IMUs) is on a footwear. This placement allows to limit the accumulation of navigation errors due to the bias drift of inertial sensors and is generally a preferable placement of sensors to achieve the highest precision of pedestrian inertial navigation. However, inertial sensors mounted on footwear experience significantly higher accelerations and angular velocities (10s of $\mathrm{g}$ and $1000 \mathrm{~s}$ of $\mathrm{deg} / \mathrm{sec}$ ) during regular pedestrian activities than during more conventional navigation tasks, which could exceed Full Scale Range (FSR) of many commercial-off-theshelf IMUs, therefore degrading accuracy of pedestrian navigation systems. This paper proposes a reconstruction filter to mitigate

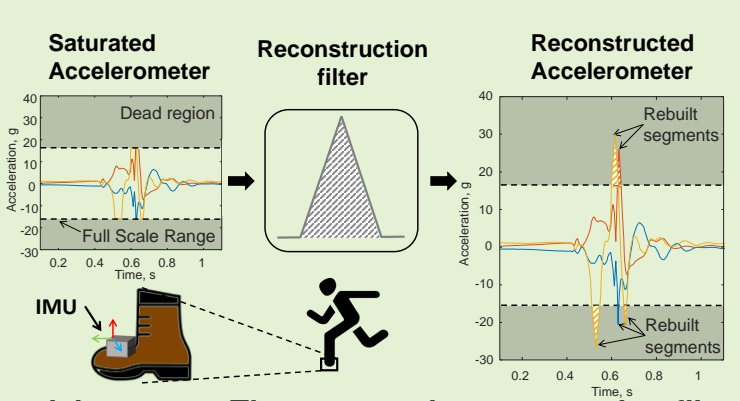
localization error in pedestrian navigation due to insufficient FSR of inertial sensors. The proposed reconstruction filter approximates immeasurable accelerometer's signals with a triangular function and estimates the size of the triangles using a Gaussian Process (GP) regression. To evaluate performance of pedestrian navigation systems enhanced by the proposed reconstruction filter, we conducted two series of indoor pedestrian navigation experiments with a VectorNav VN-200 IMU and an Analog Device ADIS16497-3 IMU, which have accelerometer's FSR of $\pm 16 \mathrm{~g}$ and $\pm 40 \mathrm{~g}$, respectively. In the first series of experiments, forces experienced by the foot-mounted IMUs did not exceed the FSRs of the sensors, while in the second series, the forces surpassed the FSR of the VN-200 IMU and saturated the accelerometer's readings. The saturated accelerometer's readings reduced the accuracy of estimated positions using the VN-200 by $1.34 \times$ and $3.37 \times$ along horizontal and vertical directions. When applying our proposed reconstruction filter to the saturated accelerometer's measurements, the navigation accuracy along horizontal and vertical directions was increased by $5 \%$ and $50 \%$, respectively, as compared to using unreconstructed signals.
\end{abstract}

Index Terms - Sensor Signal Processing, Inertial Measurement Unit (IMU), Zero Velocity Update (ZUPT), Inertial Navigation System (INS), Pedestrian Navigation, Extended Kalman Filter (EKF), Inertial Sensor Full-Scale Range (FSR), Signal Reconstruction, Indoor Localization, Gaussian Process Regression

\section{INTRODUCTION}

Thanks to the successful development of Micro-ElectroMechanical-System (MEMS) technology, Inertial Measurement Units (IMUs) with small form factors have become broadly available. Among numerous applications, the miniaturization of IMUs has enabled pedestrian Inertial Navigation Systems (INS), which utilizes IMUs mounted or attached on different parts of a human body, including head [1], pocket [2], and foot [3]-[6], and uses a combination of IMU measurements and local bio-mechanical information for positioning. The pedestrian INS operates in a self-contained manner, requires short installation time, and provides consistently available measurements [7]. These properties make the

This work is performed under the following financial assistance award: 70NANB17H192 from US department of Commerce, National Institute of Standards and Technology (NIST). Federal Program Officer Joseph Grasso.

C.-S. Jao and A.M. Shkel are with Department of Mechanical and Aerospace Engineering, University of California, Irvine, CA 92697 USA, (e-mail: chishihj, ashkel@uci.edu). pedestrian INS an attractive option to localize emergency personnel, such as firefighters or first responders, when operating in extreme environments where signals of Global Navigation Satellite System (GNSS) are degraded, visibility is limited, and information about surrounding infrastructures may not be available [8].

Among pedestrian INS solutions, foot-mounted INS have drawn attention for their ability to significantly enhance a strapdown INS using a Zero velocity UPdaTe (ZUPT) algorithm [9]. The strapdown INS performs localization by dead reckoning based on IMU measurements [10]. Due to noise and stochastic time-varying biases of MEMS-based IMUs, an accumulated navigation error of inertial navigation can exceed one meter within just a few seconds of navigation [11]. Inertial navigation can be enhanced with the ZUPT algorithm, which is designed based on the observation that velocities of a person's foot during walking are nearly equal to zero during the stance phase of a gait cycle. Using this navigation approach, the algorithm periodically resets velocity errors of the INS when the stance phase is detected [12]. The ZUPT-aided INS with 
an industrial-grade IMU has been analytically predicted and experimentally demonstrated to achieve an error of less than $1 \%$ of the traveling distance [13].

Localization accuracy of a ZUPT-aided INS has appeared to vary in different events, such as walking and running [14]. Intuitively, the difference in accuracy could be considered as a direct result of inconsistent performance of the stance phase detection in different situations [15]. Therefore, extensive research has been conducted on developing approaches, including adaptive mechanisms [16] [17], machine learning [18] [19], and sensor fusion [20] [21], to increase the detection performance. The experimental results of these studies showed that the navigation accuracy in the case of running was always worse than in the case of walking. For example, the research group in [18] demonstrated that the maximum displacement error of the ZUPT-aided INS was $1.31 \mathrm{~m}$ in the case of walking and $4.3 \mathrm{~m}$ in the case of running a distance of $130 \mathrm{~m}$. In both cases, the research group used the same IMU system carefully determined zero velocity thresholds for stance phase detection to minimize the navigation errors, yet the result of accumulated errors was different in walking-vs-running scenarios. These results indicated that there were additional error sources causing the inconsistent accuracy of ZUPT-aided INS in these different situations.

Insufficient sensor Full Scale Range (FSR) has been reported as an error source in foot-mounted INS [22]. Although mounting IMUs on footwear brings the benefits of using the ZUPT-aided INS, foot-mounted sensors experience significantly larger forces than the sensors mounted on other parts of a body. As we will show in Fig. 2 and discuss in Section II$\mathrm{A}$, the forces as large as $40 \mathrm{~g}$ can occur during the heel-strike phase of a gait cycle and saturate measurements obtained from many Commercial-Off-The-Shelf (COTS) high-performance IMUs, which have an excellent noise performance but often limited sensor FSR. There are COTS IMUs with accelerometer's FSR larger than $200 \mathrm{~g}$, which might be sufficient for footmounted INS, but these sensors are not preferable for inertial navigation because they usually have an order-of-magnitude worse noise performance. With high-performance IMUs, the saturation indicates that foot-mounted IMU could produce incorrect readings of actual accelerations during the heel-strike phases, which leads to degraded navigation accuracy and needs to be taken into account in pedestrian navigation. The trade-off between sensor's noise performance, full scale range, and Size, Weight, Power, and Cost $(\mathrm{SWaP}+\mathrm{C})$ remained to be unresolved challenges in development of sensors for inertial navigation.

In order to address this problem, previous works have attempted different approaches. In [23] and [24], shockabsorbing pads were attached between an IMU and a shoe. The pad could reduce the magnitude of forces experienced by the IMU to a certain extent, but the forces could still be larger than accelerometer's FSR of many COTS IMUs. In [25], the research group modified the ZUPT-aided INS algorithm by applying zero position change during heel strike phases and adjusting estimation error covariance matrices. Their algorithm was designed based on the assumption that displacements of an IMU mounted on the heel side of a shoe are minimal during heel strike phases. Based on our observation in pedestrian navigation experiments, we considered that the assumption might not be realistic in some scenarios, such as running, where the foot-mounted IMUs can be very unstable during the heel-strike phases. In [26], an additional IMU mounted on the calf of a human was used to assist a foot-mounted IMU. The usage of the calf-mounted IMU were shown to improve navigation accuracy but increased hardware complexity of a pedestrian navigation system.

In this paper, we propose a reconstruction filter to preprocess accelerometer signals for a ZUPT-aided INS. The proposed reconstruction filter aims to rebuild saturated accelerometer signals due to insufficient accelerometer's FSR. We consider that reconstructing saturated accelerometer signals is equivalent to predicting immeasurable signals. Immeasurable signals are defined in this paper as the acceleration outputs that can be correctly measured by accelerometers with very high FSR but are immeasurable if the FSR is low. The concept of the proposed reconstruction filter is based on observations that profiles of immeasurable signals during the heel-striking phase are similar to a triangular function, which are parameterized with amplitude $\alpha$ and period $\lambda$. When a sequence of saturated accelerometer signals are detected, the proposed reconstruction filter estimates the parameters $\alpha$ and $\lambda$, generates a triangle wave based on the estimated parameters, and superimposes the triangle wave to the saturated accelerometer signals. Reconstructed accelerometer's signals, which are outputted from the reconstruction filter, are inputted to the ZUPT-aided INS. Because noise performance of reconstructed signals and raw signals are different, the process noise settings for accelerometer bias states in the EKF need to be modified. The proposed reconstruction filter also predicts a noise variance for every reconstructed accelerometer's readings, and the predicted noise variances are used to vary the value of the process noise in the ZUPT-aided INS.

This paper makes the following contributions:

1) provides experimental evidence that COTS footmounted inertial sensors can have inaccurate accelerometer readings during the heel-strike phases due to insufficient FSR in daily pedestrian activities;

2) introduces an accelerometer's measurement model that considers effects of insufficient FSR;

3) develops a reconstruction filter to pre-process saturated accelerometer measurements for a ZUPT-aided INS;

4) verifies the proposed approach with real-world indoor pedestrian navigation experiments.

The rest of the paper is organized as follows. Section II presents a problem formulation. Section III discusses derivation and implementation of the proposed reconstruction filter. Experimental results are presented in Section IV. Finally, Section V concludes the paper with a highlight of our main results and outlook.

\section{FSR REQUIREMENTS OF FOOT-MOUNTED IMUS}

In this section, we discuss properties of IMU measurements at different mounting positions, sources that lead to incorrect readings, and profiles of saturated IMU measurements. 


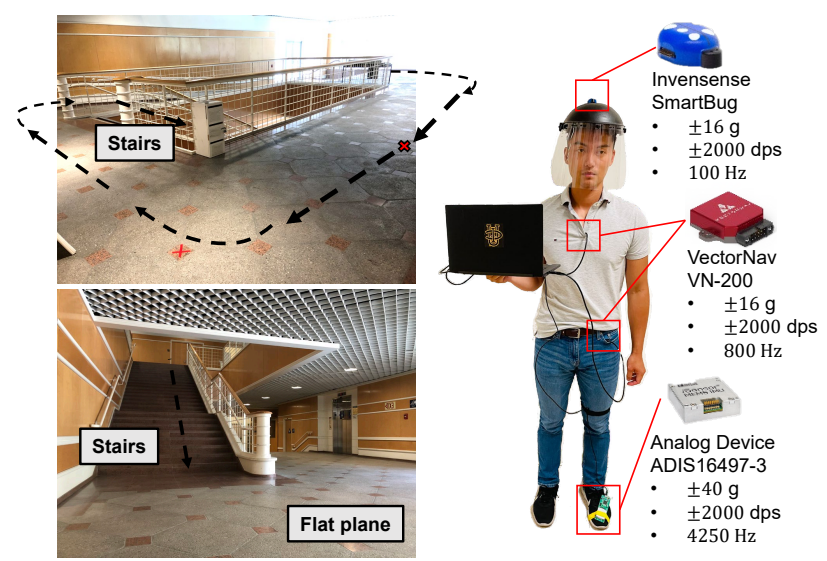

Fig. 1. Experimental setup and experiment scenario discussed in Section II-A. The Smartbug IMU (TDK/InventSense) was attached with a double-sided tape on a face shield worn by the person. One VN-200 (VectorNav) was attached with a double-sided tape on chest of a person. The other VN-200 was placed inside the left pocket. The ADIS16497-3 (Analog Devices) was mounted with tape on the toe side of the left shoe.

\section{A. IMU readings at different body mounting position}

An IMU consists of a triaxial accelerometer and a triaxial gyroscope, measuring specific forces that are proportional to linear accelerations and angular velocities experienced by the inertial sensors. During pedestrian navigation, IMUs deployed on different locations of a human body experienced different forces, which generated distinct patterns of IMU measurements.

To understand characteristics and limitations of the IMU measurements, we conducted a series of pedestrian navigation experiments of different pedestrian activities with multiple IMU mounting positions. The experiments were conducted in the Engineering Gateway Building at the University of California, Irvine. Fig. 1 shows an experimental setup and experimental environment for this study. In the experiments, four IMUs, including one Invensense SmartBug, two VectorNav VN-200, and one Analog Device ADIS16497-3, were mounted firmly, with tapes, on the head, chest, left pocket, and left shoe of a pedestrian, respectively. With this setup, the pedestrian performed nine different activities, which are listed on the y-axis label of Fig. 1. While performing the activities, the pedestrian used a metronome as a reference to the tempo of each step, not as a measurement itself. In each activity listed in Fig. 1, a slow movement corresponds to a tempo of approximately 60 steps per second, and a fast movement means 120 steps per second. Duration of each experiment was around 60 seconds.

Fig. 2 demonstrates the experimental results in the series of experiments. In Fig. 2, plots in the same column display IMU readings collected with the corresponding sensor as described in Fig. 1, and plots in the same row reveal the measurements obtained within one complete gait cycle while performing the same activity. In this paper, one complete gait cycle contains two stance phases and one swing phase. The stance phase is the period when a foot is stationary on the ground, and the swing phase is when the foot is traveling in the air. In this series of experiments, the largest angular velocities were always measured in the step in which the largest acceleration occurred. The maximum magnitudes of accelerometer's readings and gyroscope's readings collected in each experiment with the different IMUs are documented in TABLE I.

The following observation can be made about results presented in Fig. 2.

1) In most activities, except for jumping, the $z$-axis accelerometer's measurements collected by the four different IMUs exhibit two peaks. In the cases of headand chest-mounted IMUs, the peaks were generated by the heel-strike phases of two consecutive strides taken by two different feet. In the cases of pocket- and footmounted IMUs, the peaks were produced during the toeoff phase and the heel-strike phase of the same foot in the gait cycle.

2) Patterns of accelerometers' and gyroscopes' measurements collected by the four IMUs appeared different during the stance and swing phases. These differences can be utilized for step detection, as described in [1] with head-mounted IMUs, [27] with pocket-mounted IMUs, and [13] with foot-mounted IMUs.

3) Foot-mounted IMUs demonstrated a distinct characteristic, as compared to other mounting positions. For example, during the stance phases, the acceleroemters' readings were nearly identical to unit gravity and the gyroscopes' readings were very close to zero measurements. This distinct characteristic allows for robust stance phase detection. Furthermore, with the assumption that the foot has minimal motion when the foot is on the ground, the characteristic enables applying a ZUPT algorithm to the strapdown inertial navigation systems.

4) The magnitudes of linear accelerations and angular velocities experienced by the foot-mounted IMU were significantly larger than the IMUs mounted at other positions. In this series of experiments, nominal gyroscope's FSR of the four different sensors had similar values of approximately \pm 2000 degree per second (dps), which was verified by an inertial characterization equipment centrifuge Ideal Aerosmith Model 1571 at UCI Microsystems Lab [28]. It can be observed in TABLE I, that the \pm 2000 dps gyroscope's FSR was not large enough for the case of jumping. Note in TABLE I, maximum accelerometers' and gyroscopes' readings of the Analog Device IMU were larger than their nominal FSR. This is because for the Analog Device's IMU, FSR is the largest amount of acceleration the sensor can measure accurately. A measurement larger than the FSR may not reflect the actual force the sensor experienced.

5) Nominal accelerometers' FSR of the four IMUs were different, which were $\pm 16 \mathrm{~g}$ for the Invensnese SmartBug and Vector VN-200 and $\pm 40 \mathrm{~g}$ for the Analog Device ADIS16497-3. The nominal accelerometers' FSRs were confirmed by inertial characterization in UCI Microsystems Lab with shaker APS Dynamics Model APS500 [28]. In TABLE I, we can observe that the accelerometers' FSR of the SmartBug and the VN-200 were not sufficient to fully reconstruct forces experienced by the foot-mounted IMU in some activities, such 


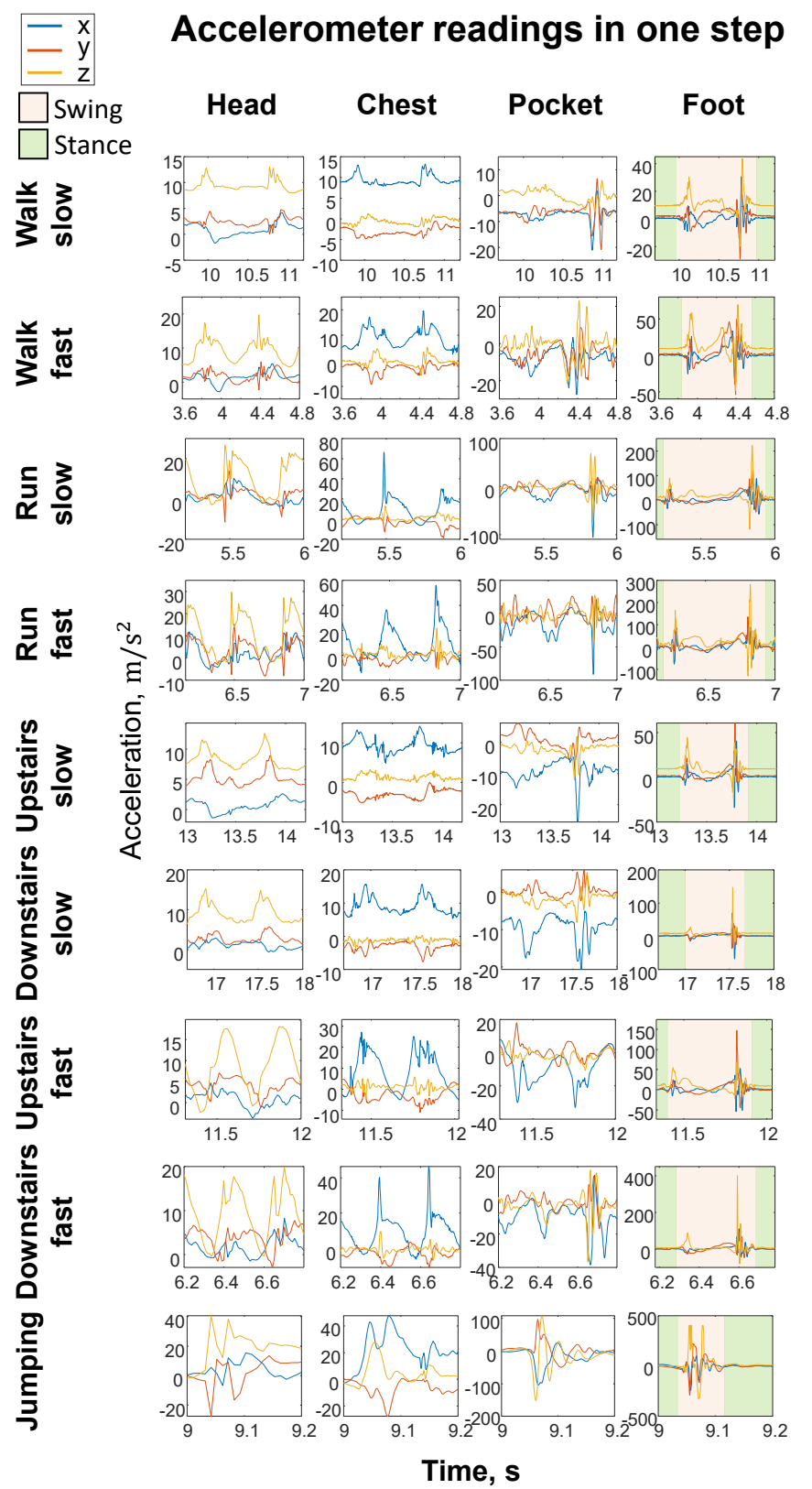

\section{Gyroscope readings in one step}

Head Chest Pocket Foot
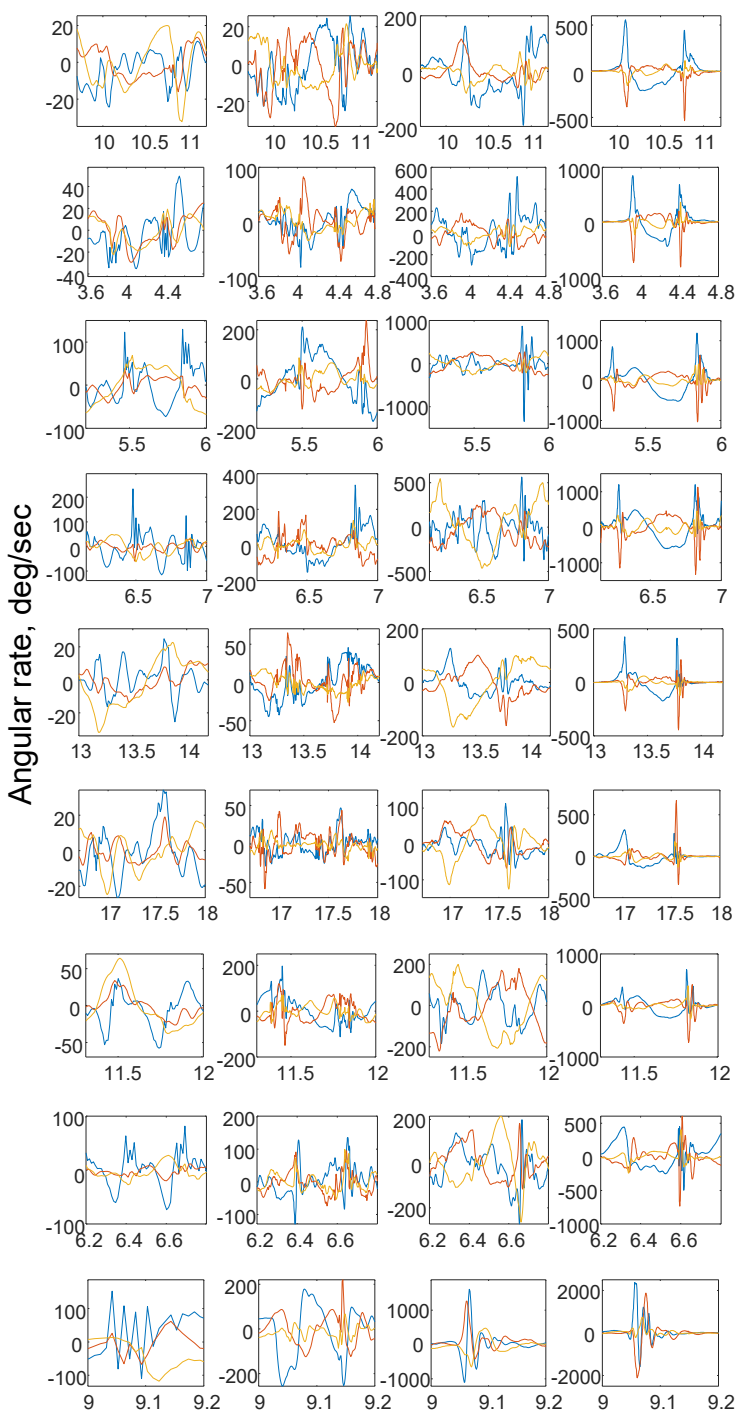

Time, s

Fig. 2. IMU readings at different mounting positions of a human body while performing everyday pedestrian activities. Plots in the same column show IMU readings collected with the same sensor, and plots in the same row correspond to measurements obtained within one complete gait cycle while performing the same activity.

as running slow, running fast, going downstairs fast, and jumping. In the cases of going downstairs fast, and jumping, even a $\pm 40 \mathrm{~g}$ accelerometer's FSR was not high enough.

Based on these IMU readings, we emphasized that although foot-mounted IMU has the advantage of being able to use the ZUPT-aided INS algorithm, the requirements of sensor's FSR for foot-mounted systems are higher, and many COTS highperformance IMUs might not have characteristics that meet the requirements of the application. In this paper, we focus on the issue of insufficient accelerometer's FSR in foot-mounted pedestrian navigation. In the next section, we demonstrate how the saturated accelerometer measurements may cause a significant error growth.

\section{B. Incorrectness of saturated IMU measurements}

As we have shown in Fig. 2, foot-mounted inertial sensors are experiencing high linear accelerations and angular velocities while performing common pedestrian activities. When sensors are not designed for the required FSR, the pedestrian activities may lead to saturated IMU measurements. To confirm that saturated measurements do not represent actual forces experienced by a foot-mounted IMU, we conducted two series of ten indoor walking experiments with two IMUs, including an Analog Device ADIS16497-3 and a VectorNav VN-200, in the Engineering Gateway Building at the University of California, Irvine. The experimental setup is shown in Fig. 3(a). The two IMUs were mounted firmly next to each other on a pedestrian's right foot, as illustrated in Fig. 3(b). The illustrated scenario will be used in the next section to 
TABLE I

MAXIMUM ACCELEROMETER'S AND GYROSCOPE'S READINGS IN DIFFERENT ACTIVITIES.

\begin{tabular}{lcccccccc}
\hline \multirow{2}{*}{ Mounting position } & \multicolumn{2}{c}{ Head (SmartBug) } & \multicolumn{2}{c}{ Chest (VectorNav) } & \multicolumn{2}{c}{ Pocket (VectorNav) } & \multicolumn{2}{c}{ Foot (Analog Device) } \\
& Accel $(\mathrm{g})$ & Gyro $(\mathrm{dps})$ & Accel $(\mathrm{g})$ & Gyro $(\mathrm{dps})$ & Accel $(\mathrm{g})$ & Gyro $(\mathrm{dps})$ & Accel $(\mathrm{g})$ & Gyro (dps) \\
\hline Walk slow & 1.4 & 51.7 & 1.4 & 58.6 & 2.3 & 214.6 & 4.4 & 586.8 \\
Walk fast & 2.1 & 71.5 & 2.1 & 126.2 & 3.8 & 576 & 14.6 & 1083.9 \\
Run slow & 3.8 & 252.6 & 7.3 & 342.3 & 11.3 & 1342.9 & 22.5 & 1182.4 \\
Run fast & 3.5 & 312.8 & 5.8 & 366.1 & 11.3 & 1922.6 & $40.9^{*}$ & $2157.3^{*}$ \\
Upstairs slow & 1.4 & 67.3 & 1.5 & 74.6 & 3.4 & 188.4 & 8 & 563.6 \\
Downstairs slow & 2.1 & 59.9 & 2.5 & 95.9 & 3.5 & 216.1 & 15 & 796.4 \\
Upstairs fast & 2.1 & 126.7 & 5.1 & 322 & 5 & 479 & 29.4 & 1047 \\
Downstairs fast & 3.3 & 195.5 & 8.3 & 245.3 & 5.3 & 553.5 & $40.8^{*}$ & 1008.6 \\
Jumping & 4.1 & 152.1 & 6.2 & 402 & 15.3 & 1617.5 & $40.9^{*}$ & $2379.2^{*}$ \\
\hline
\end{tabular}

* The measurements exceed nominal sensor FSR.

show how shortcomings of FSR of sensors can be addressed algorithmically.

In the first series of experiments, the pedestrian began by standing still for 20 seconds. Then, the pedestrian walked, in a way that he felt most comfortable, for exactly 74 steps at a pace of around 70 steps per min in a close-loop rectangular trajectory, as shown with the red lines in Fig. 3(a). During the walk, the pedestrian landed the foot on locations, marked with the red circles in Fig. 3(a), at every step. The controlled foot landing landmarks were used for better control and evaluation of the experiments. The stride length of each step was 121.92 $\mathrm{cm}$ (4 feet) when traveling along the hallway and $60.96 \mathrm{~cm}$ ( 2 feet) when perpendicular to the direction. The trajectory length was $87.8 \mathrm{~m}$, and the duration was around 120 seconds. The IMU measurements showed that, on average, a maximum acceleration shock of $2.3 \mathrm{~g}$ was generated during each step. In the second series of experiments, the pedestrian repeated the same trajectory but landed his foot hard on the floor during walking that produced a maximum shock in each step with a mean value of $23.6 \mathrm{~g}$. The maximum shock usually occurred along the vertical version. It should be noted that this acceleration exceeded the accelerometer's FSR of the VN-200. Sampling rates of ADIS16497-3 and VN-200 were set to $850 \mathrm{~Hz}$ and $800 \mathrm{~Hz}$, respectively. It is worth noting that gyroscopes' FSR, for both the ADIS16497-3 and the $\mathrm{VN}-200$, was $2000 \mathrm{deg} / \mathrm{sec}$, and the maximum angular rate

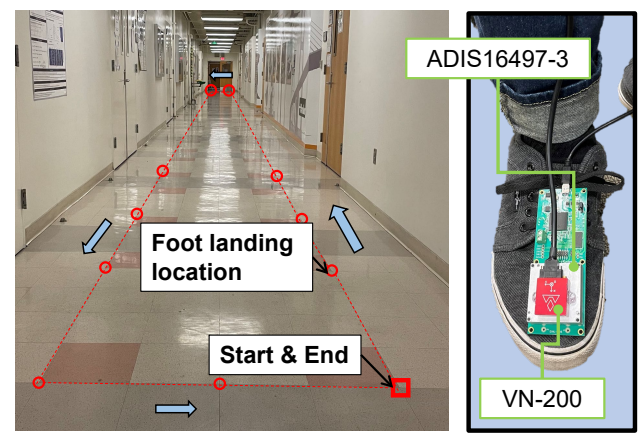

(a)

(b)

Fig. 3. (a) Experimental setup of controlled indoor navigation experiments. The red circles indicate foot-landing locations, and the blue arrows illustrate traveling directions. (b) Experimental setup of footmounted IMUs. The VN-200 IMU (red) was mounted on top of the ADIS16497-3 IMU (silver). Both IMUs were firmly attached on the toe side of the foot. measured in all the experiments was around $1600 \mathrm{deg} / \mathrm{sec}$. These angular rates were within the FSR of IMUs used for the experiments but would exceed FSR of many consumergrade COTS gyroscopes on the market.

Fig. 4 shows an example of the accelerometer's readings of the VectorNav and the ADIS16497-3 collected during one gait cycle in the first and the second series of experiments. We made three observations in Fig. 4. First, the accelerometer readings in Fig. 4(a) and (b) showed similar profiles and did not exceed the accelerometers' FSR of the two IMUs. Second, in Fig. 4(c), the Analog Device IMU measured an acceleration as large as $37 \mathrm{~g}$ during the heel strike phase, while in Fig. 4(d), the VectorNav measured a maximum acceleration of around $16 \mathrm{~g}$. Since the accelerometer's FSR of VN-200 was $16 \mathrm{~g}$, we considered that the VectorNav IMU had incorrect measurements during the second series of experiments. The highlighted area shown in Fig. 4(e) is an example of an immeasurable signal of the VN-200 IMU. Third, in Fig. 4(f), we could see that the third pick did not reach $16 \mathrm{~g}$. However, in Fig. 4(e), the Analog Device IMU clearly measured an acceleration larger than $16 \mathrm{~g}$ along the $\mathrm{x}$-axis. In our opinion, the third pick in Fig. 4(f) is an incorrect measurements, but it did not display a value as large as the accelerometer's FSR of the VN-200 because during the short period of time, measurements were not sampled by the inertial sensor when the maximum acceleration occurred.

These two series of experiments illustrated that in the case of insufficient FSR, large forces experienced by the footmounted IMU could lead to incorrect accelerometer's readings. The matching between FSR and profile of accelerometers is critical in ZUPT-aided INS. The next section of this paper will introduce a reconstruction filter that aims to mitigate the negative effect that insufficient accelerometer's FSR brings to foot-mounted INS. The introduced approach allows to relax FSR requirements.

\section{The Proposed Reconstruction Filter}

This paper aims to provide an approach to reconstruct, in real-time, accelerometer's measurements that are saturated due to insufficient FSR. This section discusses modeling the saturated accelerometer's readings and presents the a reconstruction filter resolving the saturated signals. 


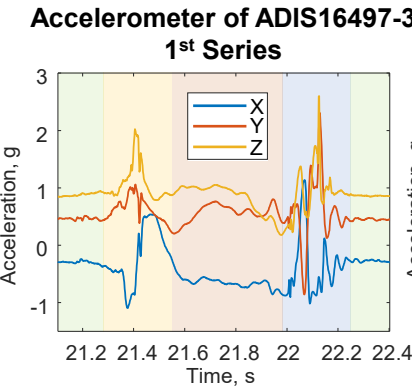

(a)

Stance phase

Accelerometer of ADIS16497-3 $2^{\text {nd }}$ Series

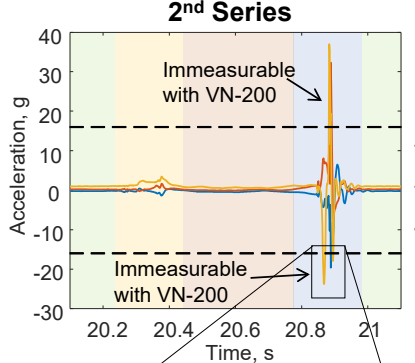

(c)

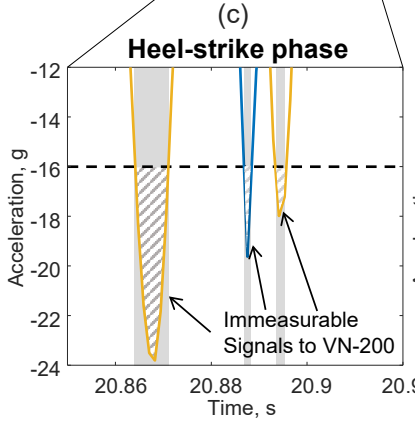

(e)

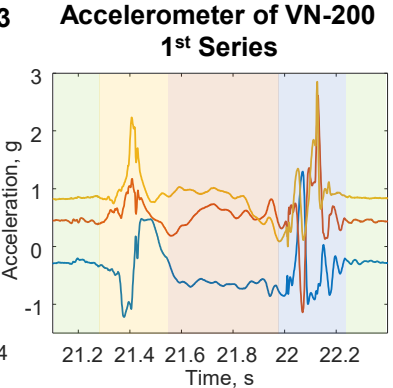

(b)
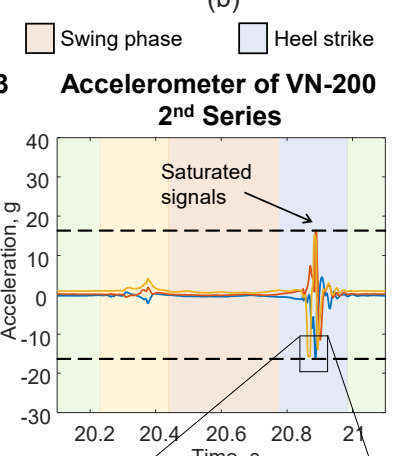

(d)

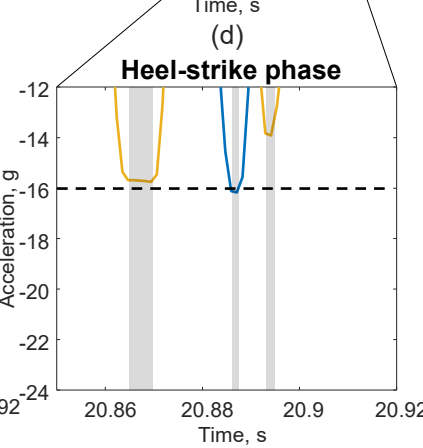

(f)
Fig. 4. (a) Accelerometer readings of one gait cycle collected with an Analog Device ADIS16497-3 IMU in the first series of experiments discussed Section II-B. (b) Accelerometer readings collected with a VectorNav VN-200 IMU during the same time period as (a). (c) Accelerometer readings of one gait cycle collected with the Analog Device IMU in the second series of experiments discussed Section II-B. Accelerometer readings collected with the VectorNav IMU during the same time period as (c). (e) A zoomed-in view of (c), showing signals pattern in a heelstrike phase of a gait cycle. The areas marked with striped patterns indicate measurements of accelerations having magnitudes larger than $16 \mathrm{~g}$. For the VN-200 IMU, these accelerometer's measurements could not be correctly measured and are called the immeasurable signals in this paper. (f) A zoomed-in view of (d), showing a saturated signal pattern in the heel-strike phase.

\section{A. Modeling of Saturated Accelerometer Signals}

This paper defines immeasurable signals of an accelerometer as the measurements of acceleration that have magnitude larger than the FSR of the sensor. Our proposed reconstruction filter is designed to predicts immeasurable signals and superimposes the predicted signals to saturated measurements. To characterize the immeasurable signals, this paper models accelerometer's measurements, $y_{a, \beta}(n)$, that are collected at time $\mathrm{n}$ with FSR of value $\beta$, as follows:

$$
y_{a, \beta}(n)=y_{F}(n)+b(n)+\omega(n),
$$

where

$$
b(n)=b_{a}(n)+\Psi_{\beta}(n), \omega(n) \sim N\left(0, \sigma_{\mathrm{VRW}}^{2}\right)
$$

Here, $y_{F}(n)$ is true specific forces experienced by the accelerometers, $\omega(n)$ is a white Gaussian noise components have a standard deviation of $\sigma_{\mathrm{VRW}}$, and $b(n)$ is an accelerometer bias. In this paper, we considered that the accelerometer bias consists of a stochastic component, $b_{a}(n)$, and a deterministic bias, $\Psi_{\beta}(n)$. The stochastic component is the inherent bias of accelerometers and is commonly described by in-run bias instability. The deterministic component $\Psi_{\beta}(n)$ is caused by using insufficient FSR of a value $\beta$ to measure large forces. The deterministic bias $\Psi_{\beta}(n)$ has the same magnitude as an immeasurable signal but with opposite sign. It is worth noting that $\Psi_{\beta}(n)$ depends on an accelerometer's FSR. Fig 4(e) and (f) show examples of profiles of accelerometer's signals collected by the Analog Device IMU and the VectorNav IMU, respectively, during a heel-strike phase. In the case of the Analog Device IMU, the accelerometer's FSR, $\beta=40 \mathrm{~g}$, and $\Psi_{40}(n)=0$ everywhere. In the case of the VectorNav IMU, $\beta=16 \mathrm{~g}$, and $\Psi_{16}(n)$ is considered to have a profiles similar to the accelerometer signals shown in Fig 4(e) that have magnitude larger than $16 \mathrm{~g}$.

It can be observed in Fig 4(e) that the immeasurable signals are formed by individual humps. We denote each of such humps as $\psi_{\beta, k}(n)$. Then, the mathematical expression of the bias $\Psi_{\beta}(n)$ can be described as follows:

$$
\Psi_{\beta}(n)=\left\{\begin{array}{lr}
\psi_{\beta, k}(n), & t\left(n_{s, k}\right) \leq t(n)<t\left(n_{s, k}\right)+\tau_{k} \\
0, & \text { elsewhere }
\end{array}\right\}
$$

Here, $t(n)$ is elapsed time at sample $n$ and $n_{s, k}$ is the sample where a hump $\psi_{\beta, k}(n)$ is first detected.

\section{B. Design of reconstruction filter}

This paper aims to provide an approach to mitigate navigation errors due to insufficient accelerometer's FSR by reconstructing the immeasurable signals. The reconstruction process is equivalent to predicting the deterministic bias component, $\Psi_{\beta}(n)$ and adding it to the saturated signals.

1) Measuring immeasurable signals: In order to study the characteristics of each $\psi_{\beta, k}(n)$, it is needed to collect a series of $\psi_{\beta, k}(n)$ during pedestrian navigation experiments. However, acquiring the true $\psi_{\beta, k}(n)$ is challenging as it would require knowledge of the true stochastic accelerometer bias, $b_{a}(n)$, which is not accessible when an IMU is in motion. In this paper, we assumed that the ZUPT-aided INS could accurately estimate the stochastic bias. Based on the assumption, we approximated $\Psi_{\beta}(n)$ and denoted the approximated version as $\Psi_{\beta}^{\prime}(n)$. Following this notation, the humps $\psi_{\beta, k}(n)$ are approximated with $\psi_{\beta, k}^{\prime}(n)$. The approximated version $\Psi_{\beta}^{\prime}(n)$ can be obtained by subtracting unsaturated accelerometer signals, collected using an IMU with a higher accelerometer's FSR, with the saturated one. For example, consider a case where one accelerometer has an FSR of value $\beta_{1}$ and another has a value $\beta_{2}$ with a condition that $\beta_{1}>\max \left(y_{a, \beta_{1}}(n)\right)>\beta_{2}$. In such case, $\psi_{\beta_{2}, k}^{\prime}(n)$ is expressed 
as follows:

$$
\begin{aligned}
\psi_{\beta_{2}, k}^{\prime}(n) & =y_{a, \beta_{1}}(n)-y_{a, \beta_{2}}(n) \\
& =y_{a, \beta_{1}}(n)-\operatorname{sgn}\left(y_{a, \beta_{2}}(n)\right) \beta_{2}, \\
& \text { for } n_{s, k} \leq n<n_{s, k}+d_{k},
\end{aligned}
$$

where $\operatorname{sgn}()$ is the sign function. The second equation holds because we assumed that during saturation periods, $y_{a, \beta_{2}}=\beta_{2}$ if $y_{a, \beta_{2}}>0$ and $y_{a, \beta_{2}}=-\beta_{2}$ if $y_{a, \beta_{2}}<0$.

In the experiments described in Section II-B, $\beta_{1}=40$ and $\beta_{1}=16$, as the the ADIS16497-3 IMU and the VN-200 IMU had an accelerometer FSR of $40 \mathrm{~g}$ and $40 \mathrm{~g}$, respectively. We used the ADIS16497-3 to collect a dataset of $\psi_{16, k}^{\prime}(n)$ for the VN-200 IMU. To do so, we artificially saturated, with a threshold of $16 \mathrm{~g}$, the accelerometers' readings collected by the Analog Device IMU in the second series of experiments described in Section II-B. In the experiments, there were 494 detected saturation events in total. Fig. 5 demonstrates an example of profiles of approximated deterministic bias $\Psi_{16}^{\prime}(n)$ for the VN-200 IMU. We could see in Fig. 5 that there were three individual humps. The yellow humps correspond to the deterministic bias along the $\mathrm{z}$-axis and the yellow hump marks the bias along the y-axis. In Fig. 5, no x-axis deterministic bias was collected. Furthermore, by observing the collected profiles of $\psi_{16, k}^{\prime}(n)$, we discovered two properties of $\psi_{16, k}^{\prime}(n)$. First, profiles of $\psi_{16, k}^{\prime}(n)$ are not identical but have a similar triangular shape. Second, we found that areas under $\psi_{16, k}^{\prime}(n)$ is proportional to saturated period.

2) Characterizing immeasurable signals: Based on the observation made in Fig. 5, we characterized each $\psi_{\beta, k}(n)$ with height, $h_{k}$, width, $\tau_{k}$, and area, $A_{k} . \tau_{k}$ is a period where a sequence of accelerometer signals are saturated and will be referred to as saturation period in the following texts. $h_{k}$ and $A_{k}$ are described as

$$
\begin{gathered}
h_{k}=\operatorname{sgn}\left(y_{a}(m)\right) \max _{n_{s, k} \leq x \leq n_{s, k}+d_{k}}\left|\psi_{\beta, k}(x)\right| \\
A_{k}=\int_{t\left(n_{s, k}\right)}^{t\left(n_{s, k}\right)+\tau_{k}}\left|\psi_{\beta, k}(t)\right| d t=\sum_{m=n_{s, k}}^{n_{s, k}+d_{k}}\left|\psi_{\beta, k}(m)\right| \delta t
\end{gathered}
$$

where $d_{k}=\frac{\tau_{k}}{\delta t}$ and $\delta t$ is the sampling period of an accelerometer.

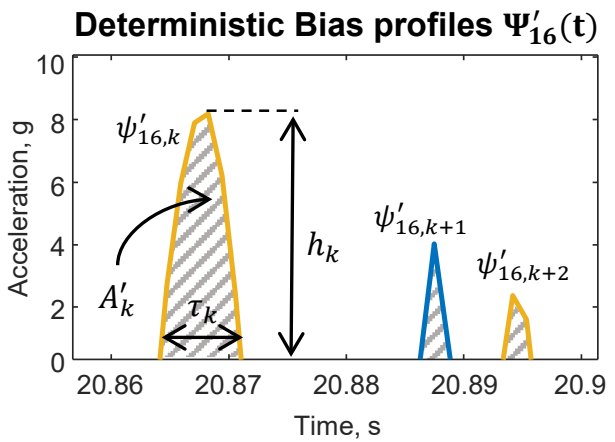

Fig. 5. Three examples of deterministic bias profiles of an IMU having accelerometer's FSR of $\mathbf{1 6} \mathrm{g}$.
In practical situation where $\psi_{16, k}^{\prime}(n)$ is used instead of $\psi_{\beta, k}(n)$, the saturation area $A_{k}^{\prime}$ can be measured as follows:

$$
\begin{aligned}
A_{k}^{\prime} & =\sum_{n=n_{s}, k}^{n_{s, k}+d_{k}}\left(\left|\psi_{16, k}^{\prime}(n)\right|\right) \\
& =\sum_{n=n_{s, k}}^{n_{s, k}+d_{k}}\left(\left|y_{a, 40}(n)-\operatorname{sgn}\left(y_{a, 40}(n)\right) 16\right|\right)
\end{aligned}
$$

Fig. 6 shows a relationship of $A_{k}^{\prime}$ and $\tau_{k}$. The data points marked in blue in Fig. 6 and their corresponding histogram indicated with the orange bar illustrate the statistics of saturation areas at different saturation periods based on the IMU measurements collected with the Analog Device IMU during the second series of experiments described in Section II-B. We could see in Fig. 6 that around $80 \%$ of the data had a saturation period of less than $6 \mathrm{~ms}$. Moreover, the statistical mode of the collected data appears at $0.0035 \mathrm{~ms}$.

3) Approximating immeasurable signals with a triangular function: Although $\psi_{\beta, k}^{\prime}(n)$ is measurable, the measurements cannot be achieved in a real-time manner if only a single IMU is used in a pedestrian navigation system. Therefore, estimation of $\psi_{\beta, k}^{\prime}(n)$ is needed. Based on the observation in Fig. 5 that the immeasurable signals have triangular shape, we hypothesized that the $\psi_{\beta, k}^{\prime}(n)$ can be approximated with a scale version of triangle wave functions, $\Lambda_{k}(n, \alpha, \lambda)$, which is expressed as follows:

$$
\Lambda_{k}\left(t, \alpha_{k}, \lambda_{k}\right)=2 \alpha_{k} \mid \frac{t}{\lambda_{k}}-\left\lfloor\frac{t}{\lambda_{k}}+\frac{1}{2}\right\rfloor,
$$

where $\alpha_{k}$ is the amplitude, $\lambda_{k}$ is the period of the triangle wave, and \lfloor\rfloor is the floor function. The area of the triangular wave, $\Delta_{k}\left(\alpha_{k}, \lambda_{k}\right)$ is calculated as

$$
\Delta_{k}\left(\alpha_{k}, \lambda_{k}\right)=\frac{\alpha_{k} \lambda_{k}}{2}
$$

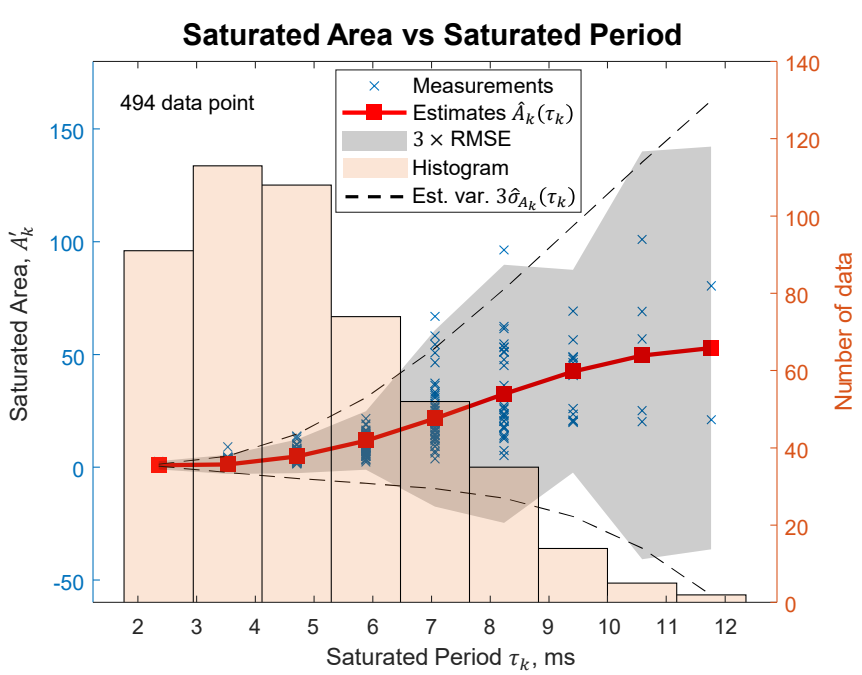

Fig. 6. Relationship of saturation area $A_{k}^{\prime}$ and saturation period $\tau_{k}$. The blue dots marked measurements of saturated area $A_{k}^{\prime}$ in the second series of experiments discussed in Section II-B. The red curve represents saturated areas predicted by a GP regression discussed in Section III-B.1. The grey shadow areas indicate the $3 \times$ RMSE of the prediction. The black dashed lines illustrate a $2^{\text {nd }}$-order polynomial for curve-fitting the RMSE. The orange bars indicate measurement distribution. 
The estimated deterministic bias, denoted as $\hat{\Psi}_{16}(n)$, is expressed as follows.

$$
\hat{\Psi}_{16}(n)=\left\{\begin{array}{lr}
\hat{\psi}_{16, k}(n), & t\left(n_{s, k}\right) \leq t(n)<t\left(n_{s, k}\right)+\tau_{k} \\
0, & \text { elsewhere }
\end{array}\right\}
$$

where

$$
\hat{\psi}_{16, k}(n)=\operatorname{sgn}\left(y_{a, 16}(n)\right) \Lambda\left(n, \alpha_{k}, \lambda_{k}\right)
$$

Here, $\lambda_{k}$ is available when operating in real-time and has the same value as $\tau_{k}$. However, $\alpha_{k}$, which determines the amplitudes of the triangle wave, cannot be measured directly from saturated accelerometer signals and needs to be estimated.

4) Estimating immeasurable signals using Gaussian Process Regression: In this paper, the estimation of $\alpha_{k}$ aims to minimize the statistical expectation of difference in $A_{k}^{\prime}$ and $\Delta_{k}\left(\alpha_{k}, \lambda_{k}\right)$. For each measured $\psi_{16, k}^{\prime}(n)$, we considered the corresponding $\tau_{k}$ as a feature and the corresponding $A_{k}^{\prime}$ as a label. Then, the saturation areas were modeled as a Gaussian Process (GP). The modeled GP has a constant basis function and a squared exponential kernel. We trained the GP with the 494 measurements of saturation areas and their corresponding saturation periods. The trained GP is found to have a parameter for the basic function, $\beta=24.5$, and an estimated noise, $\sigma=8.25$. The trained GP was then used to predict a saturation area for each detected saturation period $\tau_{k}$. The predicted saturation area is denoted as $\hat{A}_{k}\left(\tau_{k}\right)$.

The red curve presented in Fig. 6 shows the predicted saturation area $\hat{A_{k}}\left(\tau_{k}\right)$. The grey shadow area represents the RMSE of the prediction evaluated at each saturation period. Based on the results shown in Fig. 6, we made several observations. First, the RMSEs of the data corresponding to saturation period less than $6 \mathrm{~ms}$, which takes up $80 \%$ of the entire dataset, are smaller than the RMSEs of the other $20 \%$ of the data. Second, in the cases of saturated periods higher than $6 \mathrm{~ms}$, the RMSEs increased, indicating that the prediction had large uncertainties. The large uncertainties indicate that behaviors of the immeasurable signals in the cases of the higher saturation periods were less predictable. In this case, the proposed reconstruction filter can have limited performance, and sensors with a larger accelerometer's FSR are needed.

The estimation of $\alpha_{k}$, denoted as $\hat{h}_{k}\left(\tau_{k}\right)$, that minimized difference between $A_{k}^{\prime}$ in (3) and $\Delta_{k}\left(\alpha_{k}, \lambda_{k}\right)$ in (5) can be found as follows:

$$
\hat{h}_{k}\left(\tau_{k}\right)=\frac{2 \hat{A}_{k}\left(\tau_{k}\right)}{\tau_{k}} .
$$

Using (7), the height of the triangular wave function is determined, which is used to estimate $\psi_{16, k}^{\prime}(n)$.

Besides estimation of $\alpha_{k}$, we would also like to predict the uncertainty of estimated $\alpha_{k}$ because the RMSEs presented in Fig. 6 shows that the uncertainty of $\hat{A_{k}}\left(\tau_{k}\right)$ is not constant at different values of $\tau_{k}$. We used a $2^{\text {nd }}$-order polynomial with a least-square cost function to fit the calculated RMSEs marked with the grey area in Fig. 6. The fitted curve, denoted as, $\hat{\sigma}_{A_{k}}\left(\tau_{k}\right)$ was utilized to predict the uncertainty of $\hat{A}_{k}\left(\tau_{k}\right)$. The two black dashed curves shown in Fig. 6 illustrate $\hat{A}_{k}\left(\tau_{k}\right)+3 \hat{\sigma}_{A_{k}}\left(\tau_{k}\right)$ and $\hat{A}_{k}\left(\tau_{k}\right)-3 \hat{\sigma}_{A_{k}}\left(\tau_{k}\right)$, respectively.

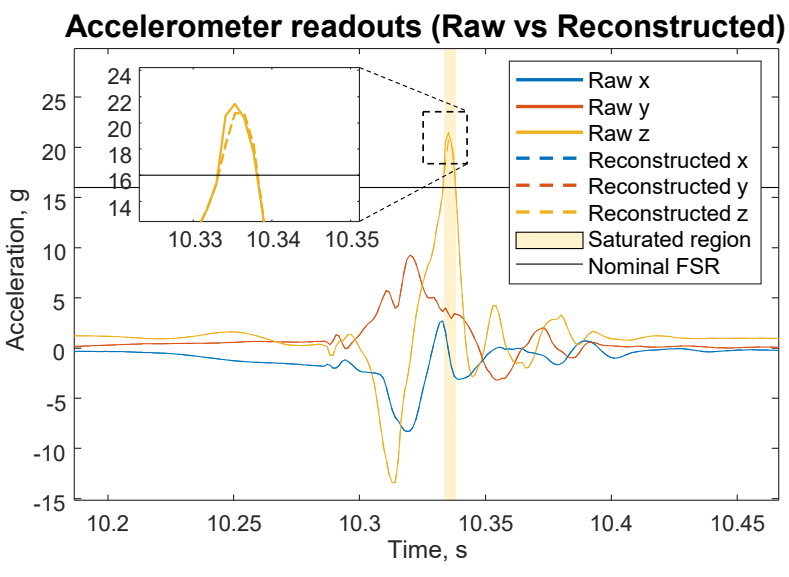

Fig. 7. Examples of raw unsaturated accelerometer's measurements collected during the heel-strike phase and artificially saturated measurements that are reconstructed by the proposed reconstruction filter.

The uncertainty of estimated $\alpha_{k}$, denoted as $\hat{\sigma}_{\alpha_{k}}\left(\tau_{k}\right)$, can be expressed as follows:

$$
\hat{\sigma}_{\alpha_{k}}\left(\tau_{k}\right)=\frac{2 \hat{\sigma}_{A_{k}}\left(\tau_{k}\right)}{\tau_{k}} .
$$

With $\hat{h}_{k}\left(\tau_{k}\right)$ in (7) and $\hat{\sigma}_{\alpha_{k}}\left(\tau_{k}\right)$ in (2), we determined that $\psi_{16, k}^{\prime}(n)$ in (6) can be best approximated with a triangular wave described as follows:

$$
\begin{aligned}
\psi_{16, k}^{\prime}(n) & \approx \hat{\psi}_{16, k}(n) \\
& =\operatorname{sgn}\left(y_{a, 16}(n)\right) \Lambda\left(n, \frac{2 \hat{A}_{k}\left(\tau_{k}\right)}{\tau_{k}}, \tau_{k}\right)+\omega_{\Lambda}(n),
\end{aligned}
$$

where

$$
\omega_{\Lambda}(n) \sim N\left(0, \hat{\sigma}_{\alpha_{k}}^{2}\left(\tau_{k}\right)\right) .
$$

5) Reconstruction of saturated accelerometer signals: In this paper, the estimated immeasurable signals $\hat{\Psi}_{16}(n)$ are used to reconstruct saturated accelerometer's readings $y_{a, 16}(n)$ when saturated signals are detected. The detection of saturated signals can be achieved by comparing accelerometer's readings with a threshold, which has a value close to a nominal accelerometer's FSR. When a saturated signal is detected, the reconstructed accelerometer's readings, $\hat{y}_{a, 16}(n)$, is expressed as follows:

$$
\begin{aligned}
\hat{y}_{a, 16}(n) & =y_{a, 16}(n)-\hat{\Psi}_{16}(n) \\
& =y_{F}(n)+b_{a}(n)+\Psi_{16}(n)-\hat{\Psi}_{16}(n)+\omega(n) \\
& =y_{F}(n)+b_{a}(n)+\hat{\omega}_{a, 16}(n),
\end{aligned}
$$

where

$$
\hat{\omega}_{a, 16}(n) \sim N\left(0, \sigma_{\mathrm{VRW}}^{2}+\hat{\sigma}_{\alpha_{k}}^{2}\left(\tau_{k}\right)\right) .
$$

Fig. 7 exhibits examples of accelerometer signals reconstructed based on the artificially saturated accelerometer signals collected with the Analog Device IMU in the second series of experiments discussed in Section II-B. To quantify the accuracy of reconstructed signals, we considered the raw unsaturated accelerometer's signals as the reference measurements. Errors of the artificially saturated signals and the reconstructed signals with respect to the raw unsaturated measurements during the saturation period were calculated. Fig. 8 
shows the error distributions of the saturated accelerometer's signals and the reconstructed signals. Few things can be noted in Fig. 8. First, the RMSE of the saturated signals was 4.63, while the RMSE of the reconstructed signals was decreased to 2.42. Second, the errors in the saturated signals had a bias of $2.97 \mathrm{~g}$, while the bias was reduced to $0.507 \mathrm{~g}$ when the reconstruction filter was used. Third, the error distribution of the saturated signals was ill-fitted with a Gaussian distribution, indicating that inputting the saturated signals to the EKF of the ZUPT-aided INS would extensively violate the assumption of the EKF. After applying the reconstructed filter, we could perceive that the shape distribution becomes closer to a Gaussian distribution. Based on the experimental result, we concluded that it was beneficial to apply our proposed reconstruction filter in foot-mounted pedestrian inertial navigation to pre-process IMU readings, which were collected with an accelerometer having an FSR of $16 \mathrm{~g}$.

\section{Use of the reconstructing filter with a ZUPT-aided INS}

The navigation framework used to produce localization solutions is a ZUPT-aided INS augmented by the proposed reconstruction filter, which is shown in Fig. 9. In the proposed framework, the ZUPT-aided INS is implemented with the SHOE detector using a constant threshold. Implementation of the ZUPT-aided INS was documented in detail in [14]. Before inputting measurements collected from a foot-mounted IMU into the ZUPT-aided INS, the proposed framework first pre-process the IMU measurements with the proposed reconstruction filter. The reconstruction filter outputs two pieces of information. The first piece is the pre-processed IMU measurements, and the second piece is the predicted uncertainty for the pre-processed measurements. The pre-processed IMU measurements are inputted to the strapdown INS and the ZUPT algorithm. The predicted uncertainty is used to boost the process noise for accelerometer bias states in the EKF.

\section{Error Distribution Before Reconstruction}

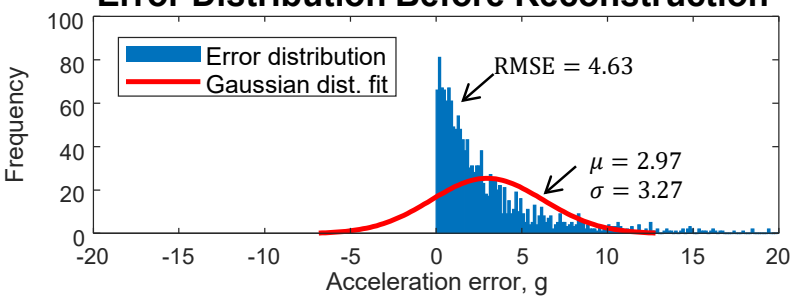

(a)

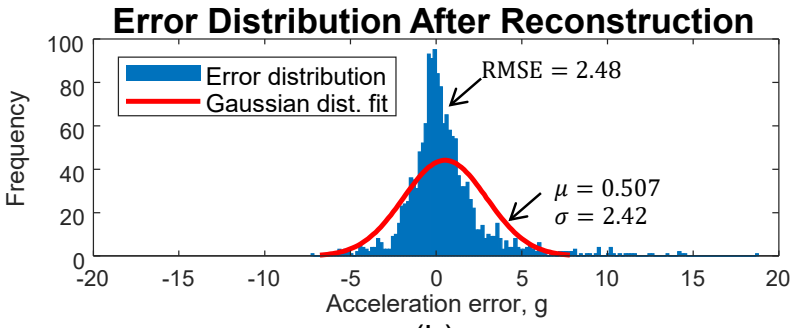

(b)

Fig. 8. (a) Error distribution of artificially saturated accelerometer's readings discussed in Section III-B.1. (b) Error distribution of the artificially saturated accelerometer's readings that were reconstructed by the proposed reconstruction filter.

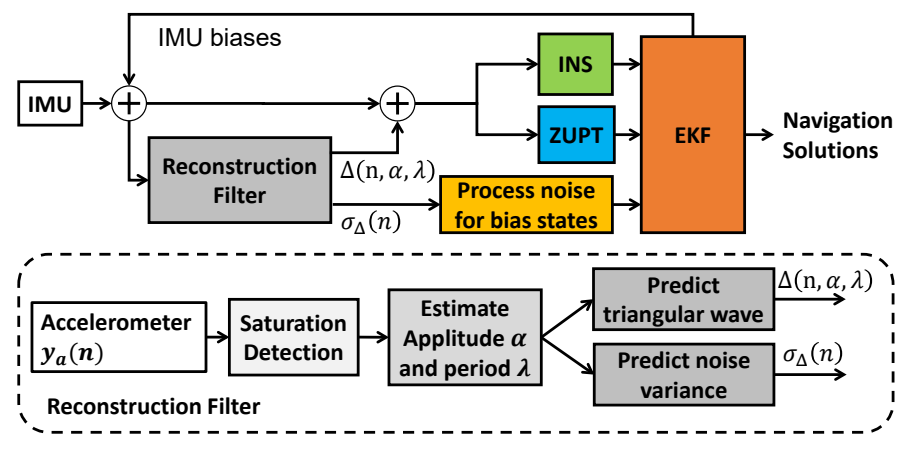

Fig. 9. Proposed navigation framework.

\section{EXPERIMENTAL VALIDATION}

We compared navigation performance of a traditional ZUPT-aided INS and the proposed ZUPT-aided INS using the reconstruction filter based on the dataset collected by the two IMUs in the two series of experiments discussed in Section II-B. In the experiments, the trajectories contained saturated sensor readings. Thresholds for stance phase detection in the ZUPT-aided INS were set to a similar value in both series of experiments, as the nominal walking paces were the same. The EKF noise parameters, including Velocity Random Walk (VRW) $\sigma_{\text {VRW }}$, Angular Random Walk (ARW) $\sigma_{\text {ARW, }}$ Rate Random Walk (RRW) $\sigma_{\text {RRW }}$, Acceleration Random Walk (AcRW) $\sigma_{\mathrm{AcRW}}$, and measurement noise for zero-velocity measurements $\sigma_{\text {ZUPT }}$ had values listed in TABLE II. In each of the experiments, initial biases of gyroscopes were estimated by taking the average of gyroscope measurements collected in the first 20 seconds when the pedestrian was standing still on the ground. During the same period, initial biases of accelerometers were estimated by the ZUPT-aided INS. The estimated initial biases were removed from the IMU measurements collected in the rest of the timestamps. The initial orientation of each experiment was determined by aligning the trajectory of the first ten steps with the north.

TABLE ॥

EKF PARAMETER SETTINGS FOR THE ZUPT-AIDED INS

\begin{tabular}{lc}
\hline EKF parameter & Value \\
\hline$\sigma_{\mathrm{ARW}}$ & $2.1597 \times 10^{-5}$ \\
$\sigma_{\mathrm{VRW}}$ & $4.8557 \times 10^{-4}$ \\
$\sigma_{\mathrm{RRW}}$ & $1.7141 \times 10^{-6}$ \\
$\sigma_{\mathrm{AcRW}}$ & $1.3873 \times 10^{-6}$ \\
$\sigma_{\mathrm{ZUPT}}$ & 0.02 \\
\hline
\end{tabular}

In Fig. 10(a), (b), (c), and (d), the blue marks show the ten trajectories estimated by the standalone ZUPT-aided INS with the two IMUs in the two series of experiments, respectively. Fig. 10(e) presents trajectories estimated by the ZUPT-aided INS using the proposed reconstruction filter based on measurements collected by the VN-200 IMU in the second series of experiments. The red circles in Fig. 10(a), (b), (c), (d), and (e) indicate the true foot-landing location in each step. The ground truth information was measured by a ruler. In Fig. 10(f), (g), (h), (i), and (j), each blue dot indicates a vector of the horizontal displacement between two consecutive steps subtracted by the ground true step-wise displacements. 

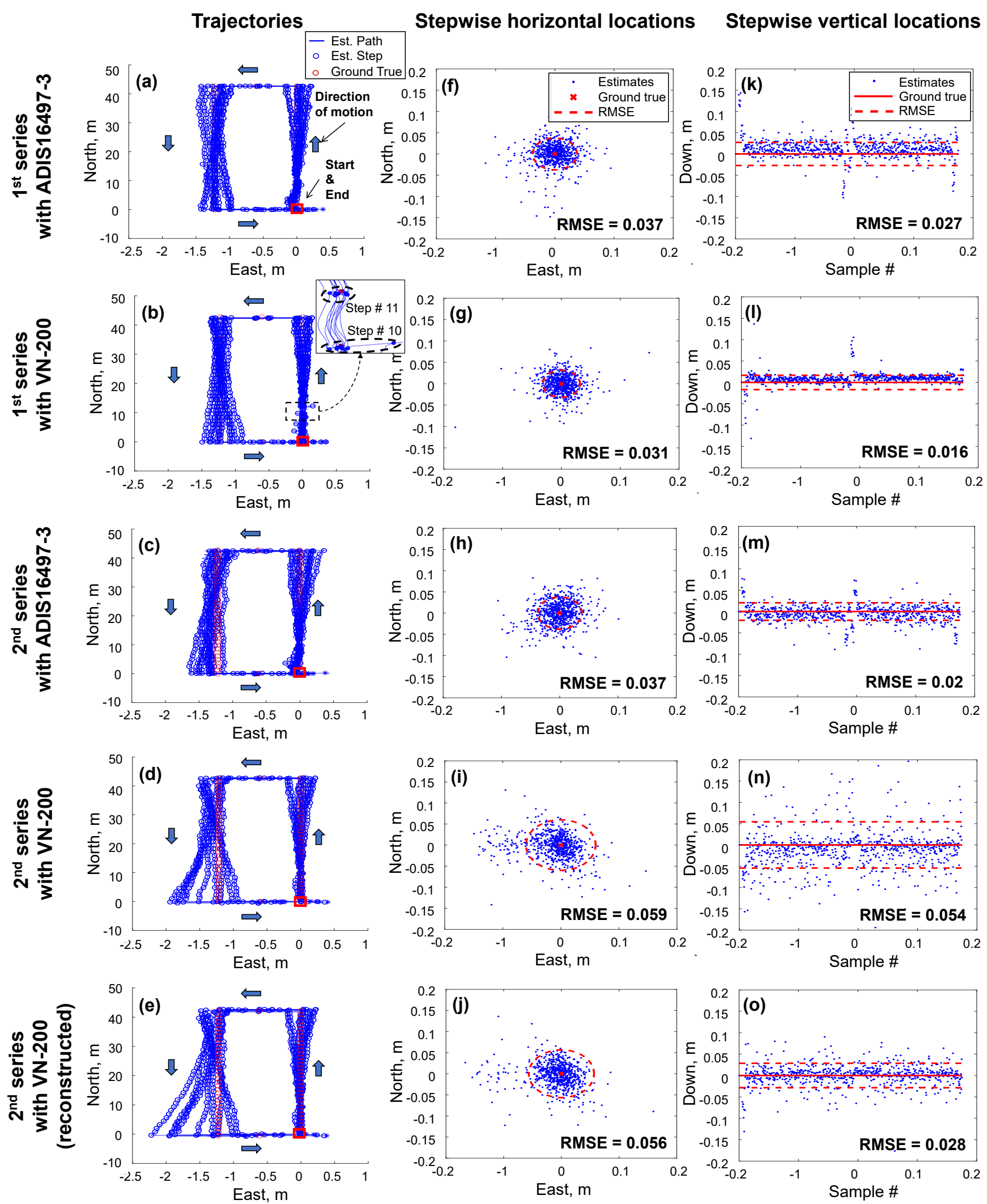

Fig. 10. (a), (b), (c), and (d) display the ground truth and the trajectories estimated by the ZUPT-aided INS using measurements collected by ADIS16497-3 and VN-200 in the first series as well as ADIS16497-3 and VN-200 in the second series, respectively. (e) presents trajectories estimated by the ZUPT-aided INS using the proposed reconstruction filter based on measurements collected by the VN-200 IMU in the second series of experiments. (f), (g), (h), and (i) present ground truth and the horizontal step-wise displacements obtained in the five approaches. The radiuses of the dashed red circles indicate the values of the corresponding horizontal step-wise Root Mean Square Errors (RMSEs). (k), (l), (m), $(\mathrm{n})$, and (o) present ground truth and vertical displacement between two consecutive steps. The red dashed lines marked the vertical step-wise RMSEs. (f), (g), (h), (i), (j), (k), (l), (m), (n), and (o) contains exactly $\mathbf{7 4 0}$ blue points. 
In Fig. 10(k), (l), (m), (n), and (o), each blue dot marks the vertical displacement between two consecutive steps. Each plot of Fig. 10(f), (g), (h), (i), (j), (k), (l), (m), (n), and (o) contains exactly 740 blue points. We computed the Root Mean Square Error (RMSE) of the step-wise displacements. In Fig. 10(f), $(\mathrm{g})$, (h), (i), and (j), the radius of the dashed red circle is the step-wise RMSE value. In Fig. 10(k), (1), (m), (n), and (o), two horizontal dashed lines represent the positive and the negative vertical step-wise RMSE. The horizontal and the vertical RMSEs are summarized in TABLE III. In TABLE III, the entries in the fourth row of the first two columns are filled as "not applicable" because no saturated measurements were detected in the first series of experiments.

TABLE III

STEP-WISE DISPLACEMENT ERRORS IN TERMS OF RMSE.

\begin{tabular}{lcccc}
\hline \multicolumn{1}{c}{ Deployed } & \multicolumn{2}{c}{ 1st series } & \multicolumn{2}{c}{ 2nd series } \\
\multicolumn{1}{c}{ sensor } & Horizontal & Vertical & Horizontal & Vertical \\
\hline ADIS16497-3 & 0.037 & 0.027 & 0.037 & 0.026 \\
VN-200 & 0.038 & 0.016 & 0.059 & 0.054 \\
VN-200 (Reconstructed) & N/A* & N/A* & 0.056 & 0.028 \\
\hline
\end{tabular}

*No saturated accelerometer's reading was detected.

A few phenomena can be noted in Fig. 10.

1) It can be observed that the step-wise navigation errors using the two different IMUs in the first series of experiments were on a similar level. Although the Analog Device IMU and the VectorNav IMU have different noise performances, the difference was negligible in our opinion and would not lead to a different navigation accuracy in a pedestrian navigation task of around 120 seconds.

2) In the second series of experiments where the two IMUs experienced large shocks, the navigation errors of the ADIS16497-3 did not increase, while the error in the case of VN-200 were $1.34 \times$ and $3.37 \times$ larger along the horizontal and vertical directions, respectively. We considered that the increased errors were caused by not only insufficient accelerometer's FSR but also insufficient accelerometer's and gyroscope's bandwidths. The consideration of insufficient bandwidth as an error source was because large shocks that occurred during the heel-strike phases generated a series of high-frequency components. These high-frequency components might exceed the accelerometer's and gyroscope's bandwidths of the VN-200, which were $260 \mathrm{~Hz}$ and $256 \mathrm{~Hz}$, respectively. Note that in the case of the ADIS164973 , insufficient sensor bandwidth was not considered as a primary error source because the ADIS16497-3 had an accelerometer's and gyroscope's bandwidths of $750 \mathrm{~Hz}$ and $550 \mathrm{~Hz}$.

3) When using our proposed reconstruction filter to preprocess the saturated measurements collected by the VN-200 IMU, the RMSEs along horizontal and vertical directions were reduced by $5 \%$ and $50 \%$, respectively. The improvements indicated that it is beneficial to preprocess IMU measurements using the proposed approach in this series of experiments. It is worth mentioning that the amount of improvements along the vertical direction was significantly larger than in the horizontal direction. In our opinion, the discrepancy was because of an observation that most of the saturated accelerometer's signals were along the $\mathrm{z}$-axis. In the $\mathrm{x}$-axis and the $\mathrm{y}$-axis, the forces experienced by the IMU saturated accelerometer's readings only a few times. As a result, the proposed reconstruction filter had limited improvements in the navigation accuracy along the horizontal direction in these experiments.

Based on the experimental results in these specific series of experiments, we demonstrated that our proposed reconstruction filter is capable to mitigate the navigation errors due to insufficient accelerometer FSR for the ZUPT-aided INS.

\section{CONCLUSION}

In this paper, we proposed a reconstruction filter for the ZUPT-aided INS. The reconstruction filter aims to rebuild the accelerometer's saturated readings due to large shocks during the heel-strike phase in a gait cycle of pedestrian navigation. We experimentally illustrated that acceleration shocks experienced by foot-mounted IMUs are an error source when an insufficient accelerometer's FSR is used, and the error cannot be ignored in pedestrian navigation. To evaluate localization performance of a ZUPT-aided INS enhanced by the proposed reconstruction filter, two series of indoor pedestrian navigation experiments were conducted with a VectorNac VN-200 IMU and an Analog Device ADIS16497-3 IMU, which have distinct accelerometer's FSRs. In the first series of experiments, the accelerometer's readings were not saturated, while in the second series of experiments, foot-mounted IMUs experienced large forces during heel-strike phases that saturated accelerometer's readings. The ZUPT-aided INS based on the two IMUs in the first series of experiments had similar navigation accuracy. In the second series, the navigation performance of the VN200 reduced by $1.34 \times$ and $3.37 \times$ along the horizontal and the vertical directions, while the position accuracy using the ADIS16497-3 remained on the same level as in the first series. When applying our proposed reconstruction filter to the saturated accelerometer's measurements, the navigation accuracy along horizontal and vertical directions was increased by $5 \%$ and $50 \%$, respectively. We draw two conclusions from the experimental results. First, the decreased performance of the VN-200 emphasized the importance of matching sensors characteristics to application requirements and, as an example, illustrated how the mismatch in the FSR of the accelerometer could degrade the performance of pedestrian inertial navigation. Second, it is beneficial to pre-process IMU measurements using the proposed reconstruction in the indoor pedestrian experiments, especially when sensors with required characteristics are not available.. The proposed approach is general and can be applied in other pedestrian navigation scenarios and applications.

\section{ACKNOWLEDGMENT}

All analytical, numerical,and experimental work was conducted in the MicroSystems Laboratory of the University of California, Irvine. 


\section{REFERENCES}

[1] S. Beauregard, "A helmet-mounted pedestrian dead reckoning system," in International Forum on Applied Wearable Computing (IFAWC), Bremen, Germany, Mar. 15-16 2006.

[2] E. Munoz Diaz, "Inertial pocket navigation system: Unaided 3D positioning," Sensors, vol. 15, no. 4, pp. 9156-9178, 2015.

[3] E. Foxlin, "Pedestrian tracking with shoe-mounted inertial sensors," IEEE Computer Graphics and Applications, no. 6, pp. 38-46, 2005.

[4] J.-O. Nilsson, A. K. Gupta, and P. Händel, "Foot-mounted inertial navigation made easy," in International Conference on Indoor Positioning and Indoor Navigation (IPIN), Busan, Korea, Oct. 27-30, 2014.

[5] S. Askari, C.-S. Jao, Y. Wang, and A. M. Shkel, "A laboratory testbed for self-contained navigation," in IEEE International Symposium on Inertial Sensors and Systems (INERTIAL), Naples, FL, USA, Apr. 1-5, 2019.

[6] C.-S. Jao, Y. Wang, Y.-W. Lin, and A. M. Shkel, "A hybrid barometric/ultrasonic altimeter for aiding ZUPT-based inertial pedestrian navigation systems," in The International Technical Meeting of the Satellite Division of The Institute of Navigation (ION GNSS+ 2020), Virtual Conference, Sep. 21-25, 2020.

[7] P. Barsocchi, S. Chessa, F. Furfari, and F. Potorti, "Evaluating ambient assisted living solutions: The localization competition," IEEE Pervasive Computing, vol. 12, no. 4, pp. 72-79, 2013.

[8] C. Fischer and H. Gellersen, "Location and navigation support for emergency responders: A survey," IEEE Pervasive Computing, vol. 9, no. 1, pp. 38-47, 2010.

[9] A. R. Jimenez, F. Seco, C. Prieto, and J. Guevara, "A comparison of pedestrian dead-reckoning algorithms using a low-cost MEMS IMU," in IEEE International Symposium on Intelligent Signal Processing, Budapest, Hungary, Aug. 26-28, 2009.

[10] D. Titterton, J. L. Weston, and J. Weston, Strapdown Inertial Navigation Technology. IET, 2004, vol. 17.

[11] M. Ma, Q. Song, Y. Li, and Z. Zhou, "A zero velocity intervals detection algorithm based on sensor fusion for indoor pedestrian navigation," in IEEE Information Technology, Networking, Electronic and Automation Control Conference (ITNEC), Chengdu, China, Dec. 15-17 2017.

[12] C.-S. Jao, Y. Wang, and A. M. Shkel, "Pedestrian inertial navigation system augmented by vision-based foot-to-foot relative position measurements," in IEEE/ION Position, Location and Navigation Symposium (PLANS), Portland, OR, USA, Apr. 20-23, 2020.

[13] Y. Wang, C.-S. Jao, and A. M. Shkel, "Scenario-dependent ZUPT-aided pedestrian inertial navigation with sensor fusion," Journal of Gyroscopy and Navigation, vol. 12, no. 1, pp. 1-16, 2021.

[14] Y. Wang and A. M. Shkel, "A review on ZUPT-aided pedestrian inertial navigation," in Saint Petersburg International Conference on Integrated Navigation Systems (ICINS), Saint Petersburg, Russia, May 25-27 2020.

[15] I. Skog, P. Handel, J.-O. Nilsson, and J. Rantakokko, "Zero-velocity detection-an algorithm evaluation," IEEE Transactions On Biomedical Engineering, vol. 57, no. 11, pp. 2657-2666, 2010.

[16] J. Wahlström, I. Skog, F. Gustafsson, A. Markham, and N. Trigoni, "Zero-velocity detection-a bayesian approach to adaptive thresholding," IEEE Sensors Letters, vol. 3, no. 6, pp. 1-4, 2019.

[17] Y. Wang and A. M. Shkel, "Adaptive threshold for zero-velocity detector in ZUPT-aided pedestrian inertial navigation," IEEE Sensors Letters, vol. 3, no. 11, pp. 1-4, 2019.

[18] B. Wagstaff, V. Peretroukhin, and J. Kelly, "Improving foot-mounted inertial navigation through real-time motion classification," in International Conference on Indoor Positioning and Indoor Navigation (IPIN), Sapporo, Japan, Sep. 18-21, 2017.

[19] Z. Chen, X. Pan, C. Chen, and M. Wu, "Contrastive learning of zerovelocity detection for pedestrian inertial navigation," IEEE Sensors Journal, pp. 1-1, 2021.

[20] C.-S. Jao, Y. Wang, and A. M. Shkel, "A Zero Velocity Detector for Foot-mounted Inertial Navigation Systems Aided by Downward-facing Range Sensor," in IEEE Sensors Conference, Virtual Conference, Oct. 25-28, 2020.

[21] M. Ma, Q. Song, Y. Gu, Y. Li, and Z. Zhou, "An adaptive zero velocity detection algorithm based on multi-sensor fusion for a pedestrian navigation system," Sensors, vol. 18, no. 10, p. 3261, 2018.

[22] J. Wahlström and I. Skog, "Fifteen years of progress at zero velocity: A review," IEEE Sensors Journal, vol. 21, no. 2, pp. 1139-1151, 2020

[23] J.-O. Nilsson, I. Skog, and P. Händel, "A note on the limitations of zupts and the implications on sensor error modeling," in International Conference on Indoor Positioning and Indoor Navigation (IPIN), Sydney, Australia, Nov. 13-15 2012.
[24] Y. Wang, S. Askari, and A. M. Shkel, "Study on Mounting Position of IMU for Better Accuracy of ZUPT-Aided Pedestrian Inertial Navigation," in IEEE International Symposium on Inertial Sensors and Systems (INERTIAL), Naples, FL, USA, Apr. 1-5, 2019.

[25] H. Ju, M. S. Lee, S. Y. Park, J. W. Song, and C. G. Park, "A pedestrian dead-reckoning system that considers the heel-strike and toeoff phases when using a foot-mounted IMU," Measurement Science and Technology, vol. 27, no. 1, p. 015702, 2015.

[26] H. Ju, J. H. Lee, and C. G. Park, "Pedestrian dead reckoning system using dual IMU to consider heel strike impact," in International Conference on Control, Automation and Systems (ICCAS), PyeongChang, GangWon Province, Korea, Oct. 17-20, 2018.

[27] D. B. Ahmed, E. M. Diaz, and S. Kaiser, "Performance comparison of foot-and pocket-mounted inertial navigation systems," in International Conference on Indoor Positioning and Indoor Navigation (IPIN), Alcalá de Henares, Madrid, Spain, Oct. 4-7, 2016.

[28] Y. Wang, Y.-W. Lin, S. Askari, C.-S. Jao, and A. M. Shkel, "Compensation of systematic errors in ZUPT-aided pedestrian inertial navigation," in IEEE/ION Position, Location and Navigation Symposium (PLANS), Portland, OR, USA, Apr. 20-23, 2020.

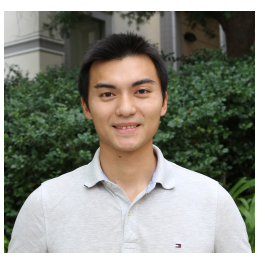

Chi-Shih Jao (S'20) received the B.S. degree in Electrical Engineering from National Tsing Hua University, Hsinchu, Taiwan, in 2015, and the M.S. degree in Electrical Engineering from Pennsylvania State University, University Park, in 2018. He is currently pursuing the Ph.D. degree with the Microsystems Laboratory, Department of Mechanical and Aerospace Engineering, University of California, Irvine, CA. His research interests include aided inertial navigation by sensor fusion approach and vision-based inertial navigation. He is a recipient of the 2019-2020 Holmes Fellowship.

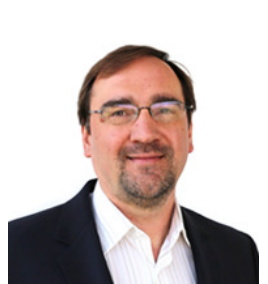

Andrei M. Shkel (Fellow, IEEE) received the diploma degree (Hons.) in mechanics and mathematics from Lomonosov's Moscow State University, Moscow, Russia, in 1991, and the Ph.D. degree in mechanical engineering from the University of Wisconsin-Madison, Madison, WI, USA, in 1997. In 2000, he joined the Faculty of the University of California, Irvine, Irvine, CA, USA, where he is currently a Professor with the Department of Mechanical and Aerospace Engineering. He has served as a Program Manager for the Microsystems Technology Office, Defense Advanced Research Projects Agency (DARPA), Arlington, VA, USA, from 2009 to 2013. His professional interests, reflected in over 270 publications and 3 books, include solid-state sensors and actuators, micro-electromechanical systems-based neuroprosthetics, sensor-based intelligence, and control theory. He holds over 42 issued patents. His current research interests include the design, manufacturing, and advanced control of high-precision micromachined gyroscopes and self-contained inertial navigation systems. He was a recipient of the 2002 George E. Brown, Jr., Award, the 2005 NSF CAREER Award, the 2006 UCI HSSoE Best Faculty Research Award, the 2009 IEEE Sensors Council Technical Achievement Award, and the 2020 UCl HSSoE Innovator of the Year Award. In 2013, he received the Office of the Secretary of Defense Medal for Exceptional Public Service. He has also served on a number of editorial boards, most recently, as an Editor for the IEEE/ASME JOURNAL OF MICROELECTROMECHANICAL SYSTEMS, the Journal of Gyroscopy and Navigation, and the Founding Chair for the IEEE International Symposium on Inertial Sensors and Systems. He is the IEEE Fellow and the President of the IEEE Sensors Council (20202021). 